(1997) Curr. Genet. 32, 24-26

\title{
Interspecies transplacement of mitochondria in yeasts
}

\author{
Milan Osuský, Jana Kissová, Ladislav Kováč
}

\begin{abstract}
Protoplasts of a respiration-deficient rho ${ }^{\circ}$ strain of Saccharomyces cerevisiae were incubated with mitochondria isolated from various respiration-competent yeast species under conditions enabling transplacement of mitochondria. Respiration-competent cybrids were selected by plating the protoplasts on agar media containing a non-fermentable energy source. The resulting cybrids contained nuclear DNA of the acceptor $S$. cerevisiae and mitochondrial DNA of the donor species, as detected by pulsed-field gel electrophoresis of chromosomes and restriction analysis of mitochondrial DNA, respectively. Successful restoration of respiration in the $S$. cerevisiae mutant was achieved by transplacement of mitochondria isolated from the following Saccharomyces species: S. bayanus, S. capensis, S. delbruckii, $S$. exiguus, S. italicus and S. oviformis.
\end{abstract}

Key words. Cybrids. Mitochondria. Transplacement. Yeasts.

\section{Introduction}

A method for an efficient transfer of isolated mitochondria into yeast protoplasts (Sulo et al. 1989) has opened a new way for studying genes that control the communication between mitochondrial and nuclear genomes by exploiting rich possibilities of yeast molecular genetics. In cultured mammalian cells, the maintenance of mitochondria in the cells seems to depend on species-specific nuclear gene products (De Francesco et al. 1980; Giles et al. 1980; Hayashi et al. 1980; Zuckerman et al. 1984). The same may be true for plants (Bonnet and Glimelius 1990). In the case of insects, the species Drosophila melanogaster could tolerate a heteroplasmic state with the coexistence in the same cells of its own mitochondria and mitochondria from another species D. mauritiana (Matsuura et al. 1989). Eventually, viable and fertile flies of $D$. melanogaster could arise that no longer possess their own mitochondrial DNA but retain the foreign mitochondrial DNA from D. mauritiana. Such cybrids could only be obtained by transplantation of germ plasm rich in mitochondria into freshly laid eggs and not by interspecies crossing owing to nuclear incompatibilities in the interspecific hybrids. A similar problem has complicated studies with yeasts, in which interspecific cell mating or protoplast fusion have been used. This can be obviated by the method of transplacement with isolated mitochondria.

In the previous work (Sulo et al. 1989), conditions for the intraspecific transplacement have ben established: mitochondria isolated from a respiring strain of $S$. cerevisiae were transfered into protoplasts of a non-respiring strain of the same species with deleted mitochondrila DNA. After regeneration of protoplasts, cybrids were obtained carrying genetic markers of the mitochondria of the donor strain and of the nucleus of the acceptor strain. The present study shows that in the cells of $S$. cerevisiae, non functional mutant mitochondria can be substituted not only by transplaced wild-type mitochondria from its own species, but also by mitochondria from other, taxonomically closely related, species of yeasts. 


\section{Materials and methods}

Yeasts. Respiration-deficient rho ${ }^{\circ}$ strain S. cerevisiae GRF $18 \rho^{\circ}(\alpha$ his 3-11,15, leu 2$3,112, \rho^{\circ}$ ) was from the previous study (Sulo et al. 1989). The following species of the genus Saccharomyces were from the collection of the Institute of Chemistry, Slovak Academy of Sciences, Bratislava: S. bayanus, S capensis, S. carlsbergensis, S. exiguus, S. heterogenicus, $S$. oviformis, $S$. willianus. The following yeasts were kindly provided by Dr. H. Fukuhara, Institut Curie, Orsay: S. chevalieri, S. italicus, S. uvarum.

Methods. In addition to those described in the previous paper (Sulo et al. 1989), the following procedures were used: Restriction analysis of mitochondrial DNA was performed according to Maniatis et al. (1982) and karyotyping by the method described by Filipp et al. (1995). Conditions of electrophoresis: $200 \mathrm{~V}$, duration of a pulse $110 \mathrm{~s}$ for the first $12 \mathrm{~h}$ and $60 \mathrm{~s}$ for additional $12 \mathrm{~h}$.

\section{Results and discussion}

Cells of the respiration-deficient rho $^{\circ}$ mutant of $S$. cerevisiae were not able to grow on the non-fermentable substrate glycerol since their mitochondria could not synthesize ATP by oxidative phosphorylation. The ability to grow on glycerol was restored, however, after the protoplasts of the mutant yeast had been preincubated with mitochondria isolated from some respiration competent species of the genus Saccharomyces indicating that the foreign functional mitochondria have substituted, in the acceptor mutant cells, the endogenous nonfunctional mitochondria. Mitochondrial DNA isolated from the resulting cybrids exhibited the same restriction patterns as did mitochondrial DNA isolated from the donor species which were different from those of mitochondrial DNA isolated from the wild-type acceptor strain (Fig. 1). As the mutant acceptor strain was rho ${ }^{\circ}$ and, accordingly, contained no mitochondrial DNA, it could not be established by this method whether the mutant mitochondria in the cells of the cybrids continued to coexist with the acquired functional mitochondria or had been diluted out and fully replaced by the transplaced mitochondria. The pulsed-field electrophoretic analysis of some of the cybrids proved that the cybrids maintained the nucleus of the acceptor strain (Fig. 2). From a number of species of the genus Saccharomyces only mitochondria isolated from the following six species substituted the non-functional mitochondria of the S. cerevisiae mutant: S. bayanus, S. capensis, S. delbruckii, S. exiguus, $S$. italicus and $S$. oviformis. A positive result with $S$. italicus was already obtained by $M$. Dóková, J. Lazowska and P. P. Slonimski (unpublished). Negative results were reached by using as donors of mitochondria other yeast genera and also the following species of Saccharomyces: S. bailii, S. calsbergensis, S. chevalieri, S. cidri, S. douglasii, S. heterogenicus, $S$. montanus, $S$. uvarum and $S$. wilianus. The genetic distance between the latter species and $S$. cerevisiae may be too large to allow the mutual compatibility of heterologuous mitochondrial and nuclear genes. Mutations in a single gene or a few genes, either mitochondrial or nuclear, may permit the compatibility and in this way an enforced coexistence and/or collaboration may be established. The possibility to identify such genes may be the principal asset of this approach. It is substantiated by the observation of Tian et al. (1993) that a change in a mitochondrial gene of S.douglasii enabled the mitochondrial genome of this species to function in a karyoductant carrying the nuclear genome of $S$. cerevisiae, while the interspecific hybrid between the wild types of these two species exhibited nucleo-mitochondrial incompatibility (Kotylak et al. 1985). 
Although as many as 41 distinct species had been classified within the genus Saccharomyces by conventional yeast taxonomy, the number was later reduced to seven (Yarrow 1984). Sixteen of those accepted by van der Waalt (1970) have been ascribeded to synonymy with $S$. cerevisiae. Later, as a result of DNA relatedness studies, Saccharomyces sensu stricto, considered to be a single species $S$. cerevisiae, has been distinguished to encompass four different species (Vaughan Martini and Kurzman 1985). The taxonomic uncertainties should not diminish the value of our approach as long as distinct molecular differences can be ascertained to exist between the donor and acceptor strains. This was already proven in the case of a cybrid between $S$. cerevisiae and $S$. capensis, which had been constructed by this method (Lazowska et al. 1992): it could be shown that an intron in mitochondrial DNA of S. capensis exhibited a different gene conversion capacity than did that of $S$. cerevisiae and the molecular basis of this difference was established, even though the species $S$. capensis has been considered by some taxonomists to be identical with $S$. cerevisiae.

Apart from the characterization of the relevant genes in extant organisms, the transplacement procedure is well suited for studies on the evolutionary descent of mitochondria and other organelles.

\section{References}

Bonnet HT, Glimelius K (1990) Cybrids of Nicotiana tabacum and Petunia hybrida have an intergeneric mixture of chloroplasts from $P$. hybrida and mitochondria identical or similar to N. tabacum. Theor. Appl. Genet. 79: 550-555

Niki J, Chigusa SI, Matsuura ET (1989) Complete replacement of mitochondrial DNA in Drosophila. Nature 341: 551-552

Hayashi J-I, Gotoh O, Tagashira Y, Tosu M, Sekiguchi T (1980) Identification of mitochondrial DNA species in interspecific cybrids and reconstituted cells using restriction endonuclease. FEBS Lett 117: 59-62

Vaughan Martini A, Kurtzman CP (1985) Deoxyribonucleic acid relatedness among species of the genus Saccharomyces sensu stricto. Int. J. Syst. Bacteriol. 35: 508-511.

Yarrow D (1984) Saccharomyces Meyen ex Reess. In: Kreger-van Rij NJW (ed) The yeasts: a taxonomic study. Elsevier, Amsterdam

Van der Walt JP (1970) Saccharomyces Meyen emend Rees. In: Lodder J (ed) The yeasts: a taxonomic study. Elsevier, Amsterdam

Lazowska J, Sczepanek T, Macadre C. Dóková M (1992) Two homologous mitochondrial introns from closely related Saccharomyces species differ by only a few amino acid replacements in their Open Reading Frames: one is mobile, the other is not. C. R. Acad. Sci. Paris 313: 37-41

Kotylak Z, Lazowska J, Hawthorn DC, Slonimski PP (1985) Intron encoded proteins of mitochondria: key elements of gene expression and genomic evolution. In: Quagriariello E, Papa, S, Palmieri F, Saccone C(eds) Achievements and perspectives of mitochondrial research. Volume II: Biogenesis. Elsevier, Amsterdam 
Tian GL, Michel F, Macadre C, Lazowska J (1993) Sequence of the mitochondrial gene encoding subunit I of cytochrome oxidase in Saccharomyces douglasii. Gene 124: 153163

Sulo P, Griač P, Klobučníková V, Kováč L (1989) A method for the efficient transfer of isolated mitochondria into yeast protoplasts. Curr. Genet. 15: 1-6

\section{Legends to Figures}

Fig. 1. Restriction patterns of mitochondrial DNA (mtDNA) of donor species and cybrids. EcoRV was used to restrict the mtDNAs. 2, mtDNA of $S$. italicus. 3, mtDNA of cybrid CIt with mitochondria transplaced from $S$. italicus. 4. mtDNA of $S$. bayanus. 5, mtDNA of cybrid CBa with mitochondria transplaced from $S$. bayanus. 6, mtDNA of $S$. capensis. 7, mtDNA of cybrid CCa with mitochondria transplaced from $S$. capensis. 8, mtDNA of $S$. oviformis. 9 , mtDNA of cybrid COv with mitochondria transplaced from $S$. oviformis. 10, mtDNA of $S$. exiguus. 11, mtDNA of cybrid CEx with mitochondria transplaced from $S$. exiguus. 1 and 12 , SYMBOL $108 \backslash f$ "Symbol" DNA, restricted with HindIII.

Fig. 2. Pulsed-field electrophoresis of parent species and cybrids. The designation of the cybrids is the same as in Fig. 1. 1, S. italicus. 2 and 3, two independent cybrids of S. italicus, CIt 1 and CIt 2. 4. acceptor strain S. cerevisiae GRF 18SYMBOL $114 \backslash$ \f "Symbol"0. 5 and 6, two independent cybrids of $S$. bayanus, CBa 1 and CBa 2. 7, S. bayanus. 